\title{
Methodological considerations for the study of multimodal signal detection
}

\author{
HAROLD STANISLAW \\ University of California, Los Angeles, California \\ and University of New South Wales, Kensington, New South Wales, Australia
}

\begin{abstract}
The predominant psychophysical methods for investigating multimodal signal detection are reviewed. It is argued that most experimental designs cannot distinguish situations in which the stimulation of one modality affects perception in another from those in which performance is affected as observers divide their attention between modalities. Furthermore, many paradigms are confounded by the effects of temporal and stimulus uncertainty. These problems also may arise in studies of unimodal signal detection. They can be avoided by presenting suprathreshold stimuli to each modality on every trial, and by administering concurrent tasks in conditions involving divided attention.
\end{abstract}

Psychophysical experiments may study unimodal perception (by presenting stimuli to a single sensory modality) or multimodal perception (by presenting stimuli to several modalities simultaneously). Of particular interest in the latter case are crossmodal effects, in which the stimulation of one modality affects perception in another, and attentional effects, in which performance declines when attention is divided among several modalities.

The literature on crossmodal and attentional effects is extensive. London's (1954) review of Soviet work alone cites over $\mathbf{5 0 0}$ studies. (For other reviews, see Bernstein, 1970; Gilbert, 1941; Klayman, 1973a; Loveless, Brebner, \& Hamilton, 1970; Ryan, 1940; and Taylor, 1974.) However, it is still not clear whether crossmodal or attentional effects exist, because previous research has produced a bewildering array of discrepant findings. This has led recent texts on multimodal perception (e.g., Marks, 1978; Walk \& Pick, 1981) to ignore the effects entirely. This is unfortunate, because crossmodal and attentional effects have serious implications, even for studies of unimodal perception. The present paper discusses these implications, reviews various methodological problems, and offers a general methodology that avoids the shortcomings of previous designs. The paper considers only signal detection paradigms, although reaction time paradigms are also useful for studying the effects.

\section{BASIC RESEARCH ISSUES}

\section{Independence}

Are simultaneous stimuli processed independently of one another? This independence question arises in studies

\footnotetext{
I thank Lynn Olzak, James Thomas, Richard Swensson, and Dominic Massaro for providing insightful comments on earlier versions of this manuscript. Requests for reprints should be addressed to Harold Stanislaw, School of Psychology, University of New South Wales, P.O. Box 1, Kensington, NSW 2033, Australia.
}

of both unimodal perception, in which the stimuli occur in the same modality (Ashby \& Townsend, 1986; Garner \& Morton, 1969), and multimodal perception, in which the stimuli occur in different modalities. In the latter case, a lack of independence can result from crossmodal effects (i.e., for a given processing strategy, responses to the stimulation of one modality vary as other modalities are stimulated) and attentional effects (i.e., for a given type of stimulation, responses vary with the number of modalities the observer attends to).

Crossmodal effects presumably reflect "hardwired" sensory interactions (e.g., summation or inhibition between modalities). Attentional effects are cognitive in nature, reflecting the observer's processing strategy. The distinction is thus between effects driven by characteristics of the stimulus and effects driven by characteristics of the observer.

Several behavioral indicators of crossmodal effects have been reported. For example, a small portion of the adult population experiences synesthesia, which usually involves the perception of colors in response to auditory stimulation (Marks, 1975). Dynamogenic effects, in which stimulation of one modality affects the sensitivity of another, constitute another type of crossmodal effect (Gilbert, 1941; Ryan, 1940). It also has been reported that auditory stimulation or deprivation can affect critical flicker frequency, visual acuity, and the intensity and duration of visual afterimages (e.g., Bross \& Zubek, 1975; Kravkov, 1930; London, 1954; Maier, Bevan, \& Behar, 1961; Maruyama, 1959; Zeitz, 1931). The elevator illusion provides yet another example. With this illusion, objects presented in zero-gravity are seen below their true physical positions, whereas the opposite effect occurs in hypergravity (Cohen, 1981).

Neurological indicators of crossmodal effects also exist. Macroelectrode recordings suggest that the evoked response to multimodal stimulation in cats and humans is not simply a linear combination of the responses to uni- 
modal stimulation (Morrell, 1965; Shipley, 1970). Microelectrode recordings in a number of species have identified cortical cells that respond to stimulation in any of several modalities, as well as cells that respond only if several modalities are stimulated simultaneously (Fishman \& Michael, 1973; Meredith \& Stein, 1983, 1985; Newman \& Hartline, 1981; Stein \& Arigbede, 1972; Wickelgren, 1971). The optic tectum of the barn owl, for example, contains cells that appear to respond more to the location of a stimulus than to its modality (Knudsen, 1983; Knudsen \& Knudsen, 1985). Similar cells may exist in the superior colliculus of the cat (Harris, Blakemore, \& Donaghy, 1980).

It is tempting to interpret these findings directly in terms of crossmodal effects. However, higher-level cognitive processes (e.g., those that are used to determine whether a seen object corresponds to a previously felt object) must also require connections between the modalities. The functional significance of crossmodal interactions at the neural level is thus unclear. The only conclusion that can safely be drawn from neurological studies is that multimodal stimulation and unimodal stimulation evoke different neurological responses. But even this conservative reading suggests that the various modalities do not operate as independent sensory systems. This provides a strong argument for studying crossmodal and attentional effects. Indeed, research on such effects led Dashiell (1939) to assail "our predecessors with their air-tight sense modalities" (p. 2) in his presidential address to the American Psychological Association.

\section{Integration}

How is information from separate modalities integrated? A common research problem, for example, is how observers respond when two modalities provide conflicting information about a single physical event (e.g., Welch \& Warren, 1980). Such work is important, but it cannot be fully interpreted without knowledge of the information that is actually available for integration. This is ultimately limited by crossmodal and attentional effects.

These effects are important even if one's primary interest is in unimodal perception. Consider a twoalternative forced choice (2AFC) visual signal detection task, in which auditory tones are presented to help observers track the observation intervals. The auditory stimuli may affect the appearance of the visual stimulia crossmodal effect. Furthermore, since both stimuli convey meaningful information, observers may divide their attention between vision and audition-an attentional effect.

Crossmodal and attentional effects are not simply nuisance factors that need to be controlled in studies of unimodal perception; they also may provide important information regarding the mechanisms underlying perception. Several authors have suggested that organisms may vary attentional factors or their sensory thresholds to maintain an optimal level of stimulation (e.g., Lawson \& Turkewitz, 1980; Schultz, 1965, p. 30; Zentall \& Zentall, 1983). Stimulation of one modality might therefore capture attention from, or affect the sensitivity of, another modality. A related issue is whether the deaf or blind can utilize, for their intact modalities, attentional resources that were previously reserved for their disabled modality.

Crossmodal and attentional effects are also relevant in human factors applications, where there is concern over the efficacy and cost-effectiveness of multimodal displays (e.g., Tzelgov, Srebro, Henik, \& Kushelevsky, 1987).

Finally, it should be noted that real-world environments present observers with a multitude of stimuli in several modalities simultaneously; the unimodal stimulus is an artifact of the perceptual laboratory. We cannot, therefore, completely understand sensory functioning until we learn how the perceptual apparatus copes with multimodal stimulation. In fact, the same issues that have been of historical interest to students of multimodal perception are now being discussed by students of unimodal perception. This is particularly true with the increasing popularity of multichannel models for both vision and audition. Interactions between channels in the same modality are analogous to interactions between different modalities. Similarly, observers may divide their attention between channels in the same modality, just as they may divide their attention between different modalities. Crossmodal and attentional effects are therefore of interest in unimodal, as well as multimodal, settings.

Both effects can be studied within a single experimental design, as will be demonstrated below. However, most studies have used one design-heteromodal stimulationto study crossmodal effects, and a different designcompound multimodal tasks-to study attentional effects. Both designs suffer from fundamental problems, which are described in the next two sections.

\section{THE METHOD OF HETEROMODAL STIMULATION}

In the method of heteromodal stimulation, observers are instructed to detect stimuli, called homomodal, in one modality, and ignore stimuli, called heteromodal, in other modalities. The crossmodal effect is the change in the detectability of homomodal stimuli as heteromodal stimuli are introduced. ${ }^{1}$

The method of heteromodal stimulation has been used most often to study the effects of auditory stimulation on visual signal detection (Davenport, 1974; Klayman, 1973a, 1973b; Maloney \& Welch, 1972; Maruyama, 1959; McGrath, 1963; Taylor, 1974; Watkins, 1964; Watkins \& Feehrer, 1965) and of visual stimulation on auditory signal detection (Klayman, 1973a; McGrath, 1963; Taylor, 1974). The comments below are directed specifically toward these efforts, although they are equally applicable to other studies utilizing heteromodal stimulation (e.g., Benedetti \& Loeb, 1972; Bross, 1979; Bross \& Zubek, 1975; Lewkowicz \& Turkewitz, 1981; Zubek, 1969).

Many studies of crossmodal effects use performance measures, such as the proportion of signals detected, that are sensitive to changes in criteria. This is unfortunate, 
because observers may regard heteromodal stimuli as distracting (e.g., Hartmann, 1933) and therefore alter their criteria in anticipation of a performance decrement. This problem is compounded by the common practice of administering trials with and without heteromodal stimulation in separate blocks, which permits observers to adopt different criteria and/or processing strategies for the two conditions. The present discussion assumes that trials with and without heteromodal stimulation are intermixed, so that observers cannot anticipate heteromodal stimulation. It is also assumed that criterion-free measures of performance are used. These precautions eliminate changes in criteria and processing strategies as potential sources of variation.

A typical design might compare performance on a visual signal detection task given silence with performance given white noise, pure tones, music, and so forth. Such a design is depicted in Figure 1, where the area under the receiver-operating characteristic (ROC) curve serves as a criterion-free performance measure. (Identical designs apply for other criterion-free measures.) An observer is presented with trials in which a visual signal is either absent (the "noise"' trials in Figure 1) or present (the "signal" trials in Figure 1). The trials are presented in silence (top portion of Figure 1), or with constant auditory stimulation (bottom portion of Figure 1). On each trial, the observer must decide whether a visual signal was presented, perhaps combining this with a confidence rating. These responses are used to construct an ROC curve (see Green \& Swets, 1974, pp. 30-52, for details), from which the ROC area can be derived. This area reflects the observer's ability to discriminate the noise from the signal trials. The ROC areas given silence and auditory stimulation are compared to determine whether a crossmodal effect is present.

When the methodology illustrated in Figure 1 has been used in conjunction with meaningless auditory stimuli, no consistent findings have emerged. For example, consider three studies that examined the effect of auditory stimulation on performance in a $4 \mathrm{AFC}$ visual signal detection task. In one study (Watkins, 1964), performance was worse given silence than it was given broadband auditory noise. The same author later found no difference between performance given silence and that given broadband noise (Watkins \& Feehrer, 1965). A third study (Maloney \& Welch, 1972) found that performance given silence was actually better than performance given a constant, 1000 $\mathrm{Hz}$ tone.

The results are more consistent when meaningful auditory stimuli (e.g., music or real-world sounds) are used. In such studies, performance tends to improve with auditory stimulation (e.g., Davenport, 1974; Davies, Lang, \& Shackleton, 1973; McGrath, 1963), a result probably due more to arousal than to crossmodal effects. Psychophysical experiments are usually monotonous, and performance often declines over the course of an experimental session. Meaningful auditory stimuli can induce arousal, thus helping to reduce or delay this performance decrement. Indeed, an apparent crossmodal effect in a vigilance task may disappear if observers are alerted prior to the presentation of the heteromodal signal (Benedetti \& Loeb, 1972).

Auditory stimuli may be presented intermittently, rather than constantly (Figure 2). The intermittent stimuli may occur only on signal trials (top of Figure 2), or on both signal and noise trials (bottom of Figure 2). In either case, performance is usually better than it is given silence (middle of Figure 2; see Bernstein, 1970; Klayman, 1973a; Loveless et al., 1970, Experiment 4; Taylor, 1974; Taylor \& Campbell, 1976; Watkins, 1964; Watkins \& Feehrer, 1965). An obvious problem in these studies is that if heteromodal stimuli are presented only in conjunction with homomodal stimuli (top portion of Figure 2), observers may entirely ignore the homomodal stimuli and concentrate instead on detecting the heteromodal stimuli. Thus, performance may improve with heteromodal stimulation merely because heteromodal stimuli are easier to detect than are homomodal stimuli. Even if this were not the case, stimuli in the top portion of Figure 2 provide

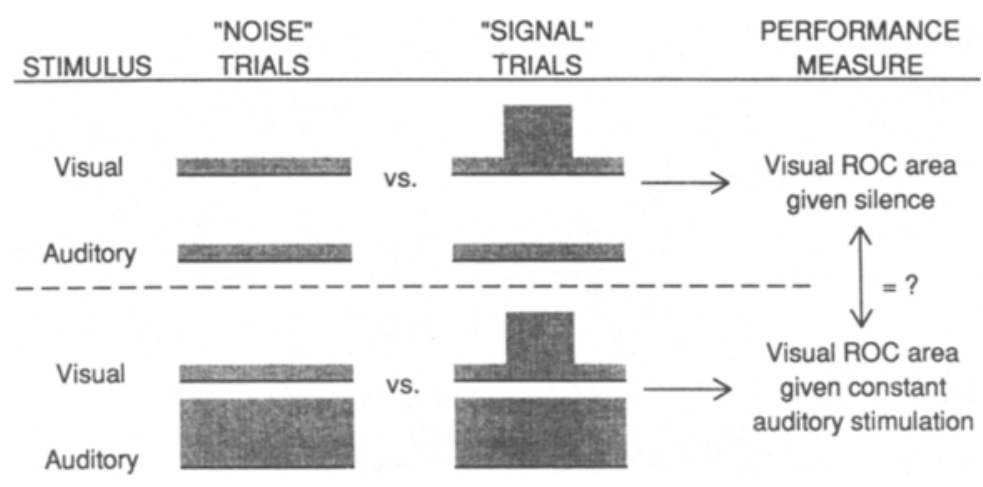

Figure 1. Stimulus pairs for an experiment involving constant heteromodal stimulation. Four pairs are depicted, with time along the abscissa and stimulus intensity along the ordinate. A comparison of responses to the noise and signal stimuli yields the receiver-operating characteristic (ROC) area, which is a criterion-free measure of signal detectability. In the absence of overt stimulation, each modality is exposed to the background stimulation level, as in the upper noise trial. 


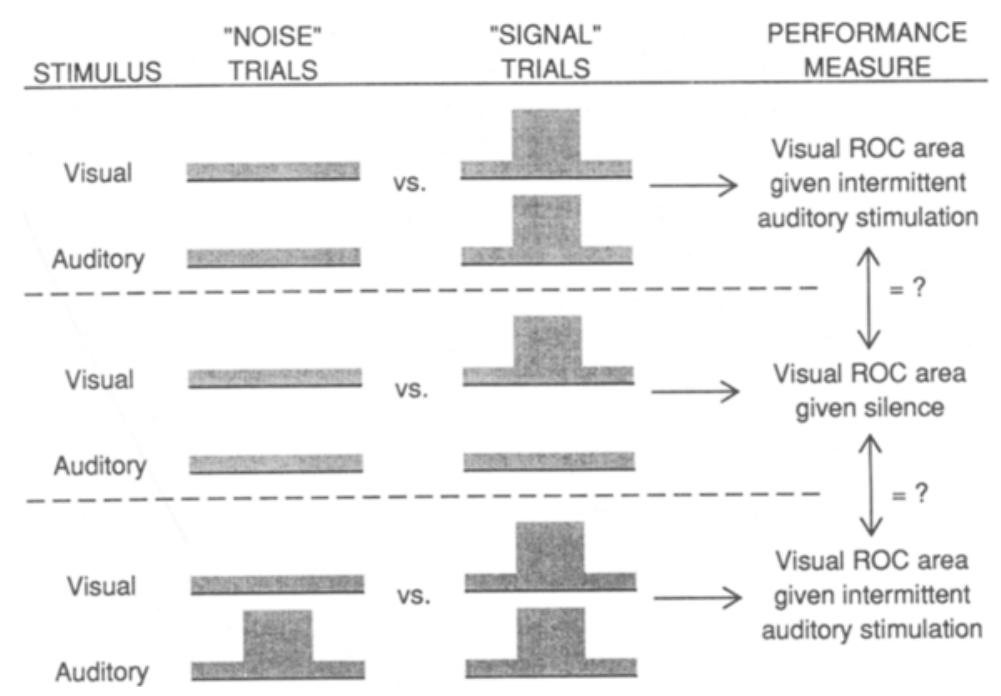

Figure 2. Stimulus pairs for an experiment involving intermittent heteromodal stimulation.

observers with two opportunities to detect a signal (one per modality), whereas stimuli in the middle portion of Figure 2 provide observers with only one such opportunity.

Clearly, there should be no correlation between heteromodal and homomodal stimulation. However, even if this precaution is taken (bottom portion of Figure 2), heteromodal stimuli may improve performance by providing information concerning the timing of homomodal stimuli. It is well known that performance declines with increasing temporal uncertainty (Green \& Swets, 1974, pp. 265-268; Kinchla, 1969). This was demonstrated by Egan, Greenberg, and Schulman (1961, Experiment 1), who found that observers were far better at detecting tones when the tone always occurred immediately after the onset of a given trial $\left(d^{\prime}=1.5\right)$ than when it could occur at any time within $8 \mathrm{sec}$ of the trial onset $\left(d^{\prime}=0.75\right)$. Thus, differential performance in the bottom two conditions of Figure 2 may simply reflect a difference in temporal uncertainty: The onset time of the homomodal stimulus is known more precisely in the bottom portion of Figure 2 than it is in the middle portion of Figure 2. In fact, several studies (e.g., Klayman, 1973a, 1973b; Taylor, 1974) demonstrated that the magnitude of the apparent crossmodal effect varies with the temporal relationship between the onset of the homomodal and that of the heteromodal stimuli.

The effects of temporal uncertainty are not readily factored out. Consider, for example, studies that require observers to detect auditory stimuli with and without an accompanying visual cue (see Klayman, 1973a, for a review). The visual cue generally improves performance, if it precedes the auditory stimulus by $0-500 \mathrm{msec}$. An obvious way to determine whether this effect stems only from the reduction in temporal uncertainty afforded by the visual cue is to measure performance given an auditory cue. The goal here is to distinguish cuing effects (which can be generated by both heteromodal and homomodal stimuli) from crossmodal effects (which can be generated only by heteromodal stimuli). If the visual and auditory cues differ in effectiveness, this could be taken as evidence of a crossmodal effect. However, the same result could arise, in the absence of a crossmodal effect, if the auditory cue masks the auditory signal. Since there is no a priori basis for deciding which cue provides an appropriate baseline for performance, ambiguity results. Furthermore, the two cues could impose different attentional loads. For example, the auditory signal might be processed in the same attentional channel as the auditory cue, but in a different attentional channel from the visual cue. Performance given a visual cue could then exceed performance given an auditory cue, but such an effect would have an attentional, rather than a crossmodal, locus. There is thus no basis for the notion that effects uncovered with the method of heteromodal stimulation are crossmodal in origin. In fact, the method can introduce complicating factors, such as reduced temporal uncertainty.

Many of the difficulties associated with the method can be avoided merely by presenting suprathreshold heteromodal stimuli on every trial, and varying the magnitude or type of stimulation instead of its presence or absence (Figure 3). An additional advantage of this modification is that conditions vary quantitatively, not qualitatively, with respect to heteromodal stimulation. This should discourage observers from "compensating" for heteromodal stimulation and varying their criteria or processing strategies. Thus, any effects that are found will most likely be crossmodal in origin.

Unfortunately, this method cannot examine attentional effects, since it presumably induces observers to adopt a consistent processing strategy across all conditions. Furthermore, it provides no indication of the degree to which heteromodal stimuli are processed, or the manner by 


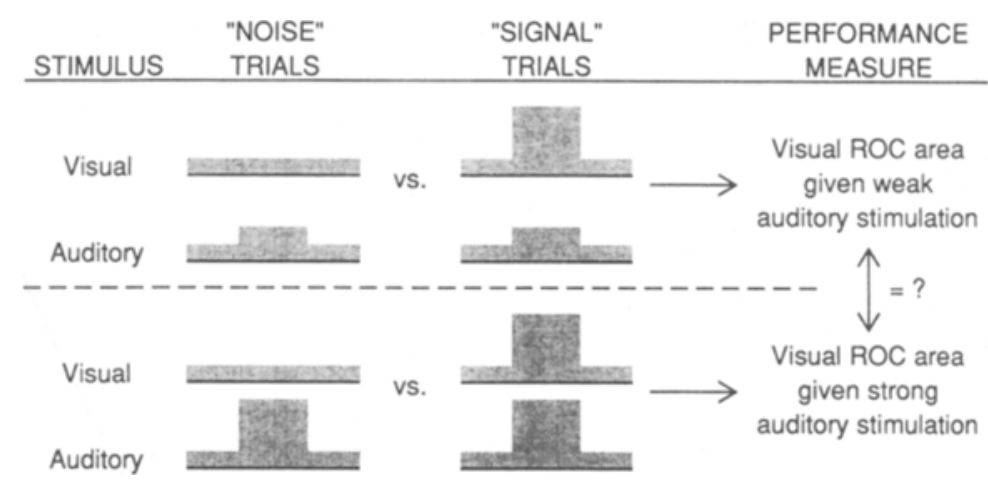

Figure 3. Stimulus pairs for an experiment involving intermittent heteromodal stimulation, modified to eliminate temporal uncertainty effects. Both levels of overt auditory stimulation are above threshold.

which attention is divided between the various modalities. To investigate these issues, the experimenter must require observers to monitor two (or more) modalities simultaneously, as described in the following section.

\section{MULTIMODAL TASKS}

Attentional effects are generally studied by asking observers to monitor several modalities simultaneously. In a compound multimodal task, a single response is used to report all signals. In a concurrent multimodal task, separate responses are used to report signals in each modality (Sperling \& Dosher, 1986).

\section{Compound Tasks}

Compound multimodal tasks have been used primarily to study the effects of dividing attention between vision and audition. In discussing these studies, an auditory noise stimulus is represented with $A_{N}$, and an auditory signal with $A_{s}$. Similarly, $V_{N}$ and $V_{S}$ represent visual noise and visual signal stimuli, respectively. Four possible stimulus pairs (e.g., $A_{N} V_{N}$ ) can then be presented.

A typical experiment utilizing compound multimodal tasks consists of unimodal visual, unimodal auditory, and bimodal conditions. The unimodal/bimodal distinction is based on the number of modalities the observer must monitor, not on the number of stimuli or responses. The unimodal visual condition provides an estimate of signal detectability when observers attend only to vision, and involves the presentation of $A_{N} V_{N}$ on noise trials and $A_{N} V_{S}$ on signal trials (as in the top portion of Figure 1). A measure of signal detectability is derived by comparing the responses to these two stimuli. Similarly, the unimodal auditory condition involves the presentation of $A_{N} V_{N}$ (noise) and $A_{S} V_{N}$ (signal), yielding an estimate of auditory signal detectability when observers attend only to audition. The bimodal condition involves the presentation of $A_{N} V_{N}$ (noise) and $A_{S} V_{S}$ (signal), as in the top portion of Figure 2. This provides an estimate of the combined detectability of auditory and visual signals when observers attend to both modalities simultaneously.
Under this paradigm, attentional effects are implicated whenever the detectability of signals in the bimodal condition deviates from a predicted value, which is based on the detectability of signals in the two unimodal conditions (for details, see Fidell, 1970; Green \& Swets, 1974, pp. 235-275). Performance in the bimodal condition usually falls short of this prediction (e.g., Brown \& Hopkins, 1966; Buckner \& McGrath, 1961; Colquhoun, 1975; Craig, Colquhoun, \& Corcoran, 1976; Gruber, 1964; Osborn, Sheldon, \& Baker, 1963), although rare exceptions have been noted (Dougherty, Jones, \& Engel, 1971; Fidell, 1970; Loveless et al., 1970, Experiment 1). These failures could signify inadequacies in the prediction equations, but they are more commonly taken as evidence for attentional effects.

Such a conclusion is premature, because the unimodal conditions lack heteromodal stimulation: $V_{S}$ is never presented in the unimodal auditory condition, and $A_{S}$ is never presented in the unimodal visual condition. Thus, there is reduced temporal uncertainty in the bimodal condition, where both modalities are stimulated on signal trials. This problem may not seem serious, as it presumably introduces a conservative bias: Attentional effects should be harder to find. Unfortunately, the situation is not nearly this simple. Crossmodal effects may produce differences between the visual percepts generated by $A_{N} V_{S}$ (the signal stimulus in the unimodal visual condition) and $A_{S} V_{S}$ (the signal stimulus in the bimodal condition), and between the auditory percepts generated by $A_{S} V_{N}$ and $A_{S} V_{S}$. The prediction equations assume constant visual and auditory percepts across conditions, and may thus fail due to crossmodal, as well as attentional, effects.

Such confounding of effects is typical of designs incorporating compound multimodal tasks. Crossmodal effects can be ruled out only by comparing performance on heteromodal noise and signal trials in each attentional condition, as described in the preceding section. However, no such comparison is possible in the bimodal condition, where the test for a crossmodal effect on vision compares the ability to distinguish $A_{N} V_{N}$ from $A_{N} V_{S}$ with the abil- 
ity to distinguish $A_{S} V_{N}$ from $A_{S} V_{S}$. $A_{S} V_{N}$ and $A_{S} V_{S}$ are both signals in the bimodal condition, and require identical responses.

If no crossmodal effects are found in either unimodal condition (where a test for such effects is possible), one might be tempted to dismiss the possibility of crossmodal effects in the bimodal condition (where no such test is possible). But it could well be the case that there is an interaction between crossmodal and attentional factors (see below).

Fidell (1970) attempted to correct for the confounding of crossmodal and attentional effects in a different manner. He found the probability of detecting the $A_{N} V_{S}$ signal in the bimodal and unimodal visual conditions, using $A_{N} V_{N}$ as the noise stimulus. Similarly, he found the probability of detecting $A_{S} V_{N}$ in the bimodal and unimodal auditory conditions, with $A_{N} V_{N}$ again serving as noise. Performance in both modalities was worse in the bimodal than in the corresponding unimodal condition. McGrath (1965) reached the same conclusion in a study of vigilance behavior, and similar findings are evident in data reported by Loveless et al. (1970, Experiment 3 ).

These results cannot be attributed to crossmodal effects, since the noise and signal stimuli were identical in the conditions being compared. They might, therefore, reflect attentional effects. However, as Fidell (1970) conceded, they might also reflect stimulus uncertainty. Even in the absence of attentional limitations, observers should suffer a performance decrement in the bimodal relative to the unimodal condition, because the former condition involves two observations (one per modality), whereas the latter involves only one observation. As the number of observations increases, there is a corresponding increase in noise, and performance suffers accordingly (Kinchla, 1969; see Sperling \& Dosher, 1986, for a detailed treatment).

This argument might seem to conflict with the earlier statement that detection is better when two signals are presented (as in the top portion of Figure 2) instead of only one (middle portion of Figure 2). That earlier statement was correct, but a problem arises when the observer does not know whether the signal will be $A_{N} V_{S}, A_{S} V_{N}$, or $A_{S} V_{S}$. If $A_{S} V_{S}$ is the only possible signal, observers can adopt criteria that will optimize the detectability of $A_{S} V_{S}$; however, if observers must also be aware of $A_{N} V_{S}$ or $A_{S} V_{N}$ (to which they must also respond "signal"), they must modify their criteria accordingly, which will impair their ability to detect $\mathrm{As}_{\mathbf{S}} \mathrm{V}_{\mathbf{s}}$.

This problem is illustrated in Figure 4, which maps auditory and visual percepts in a two-dimensional space. The intensity of the auditory percept varies along one dimension of the space, while the intensity of the visual percept varies along the other dimension. Each presentation of a stimulus pair yields a percept that can be represented as a point somewhere in this two-dimensional space. The percepts for a given stimulus pair tend to cluster about a particular mean value, but vary somewhat about this mean due to stochastic factors. This is represented by the circles in Figure 4, which are isodensity contours for the perceptual distributions associated with each stimulus pair. The distributions are largely overlapping for thresholdlevel stimuli, but are shown as distinct in the figure to improve clarity.

Keeping this overlap in mind, suppose that observers are asked to detect signals. If $A_{N} V_{N}$ and $A_{S} V_{S}$ are the only two possible stimulus pairs, observers may decide to respond "signal" whenever a percept falls above and to the right of the solid diagonal line in Figure 4. But if $A_{N} V_{S}$ and $A_{S} V_{N}$ are also presented, observers may adopt the criteria shown as broken lines in Figure 4; that is, they may decide that a visual signal was presented if the percept falls to the right of the vertical broken line, and that an auditory signal was presented if the percept falls above the horizontal broken line. They will then respond "signal" whenever the percept falls above and/or to the right of the broken lines. Now, consider what happens if $A_{N} V_{N}$ is presented, and the percept happens to fall in the shaded region of Figure 4. When $A_{N} V_{N}$ and $A_{S} V_{S}$ are the only two possible stimulus pairs, observers can use the solid line criterion, and correctly identify the stimulus as noise. However, when all four stimulus pairs are possible, observers may use the dotted line criteria, and incorrectly identify the stimulus as a signal. Performance is thus impaired when $A_{N} V_{S}$ and $A_{S} V_{N}$ are potential signals. This is the essence of the stimulus uncertainty problem. The effects of stimulus uncertainty were demonstrated by Davis and Graham (1981), who asked observers to detect visual gratings. One observer dropped from $92 \%$ to less than $70 \%$ correct when the grating could have several spatial frequencies instead of only one.

Fidell (1970) attempted to circumscribe the stimulus uncertainty problem by estimating sensitivity $\left(d^{\prime}\right)$ from the bimodal condition, rather than from the appropriate unimodal condition. In this study, auditory sensitivity was found by comparing the responses to $A_{N} V_{N}$ with those

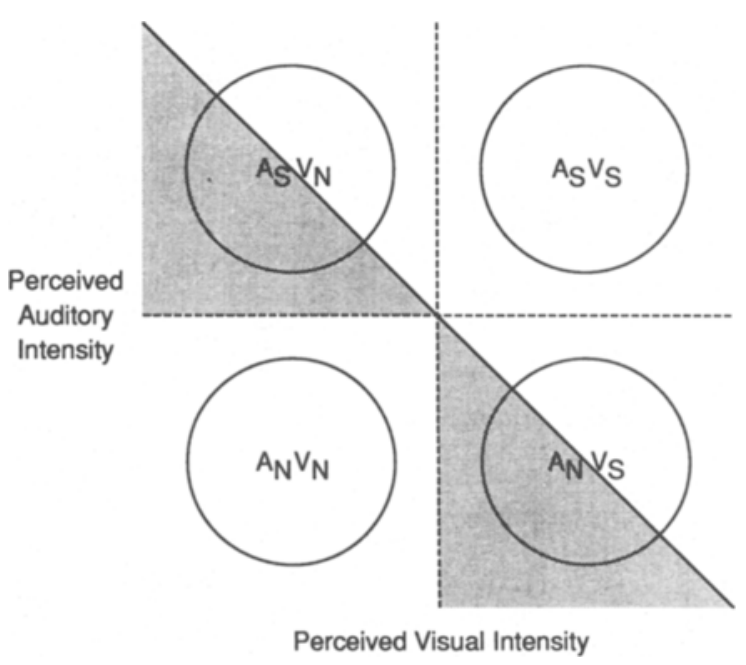

Figure 4. Criterion lines and the distribution of perceived auditory and visual intensity for four stimulus pairs. See text for details. 
to $A_{S} V_{N}$, whereas visual sensitivity was found by comparing the responses to $A_{N} V_{N}$ with those to $A_{N} V_{S}$. These values led to a predicted sensitivity for combined audiovisual signals, which was similar to the actual sensitivity (found by comparing the responses to $A_{N} V_{N}$ with those to $\left.A_{S} V_{S}\right)$. Fidell therefore concluded that attentional effects were absent. However, since all sensitivity estimates were made in the bimodal condition, this approach does not test for attentional effects at all; the comparison is not between conditions that differ in their attentional load. Instead, the comparison is between conditions with different levels of heteromodal stimulation: Auditory and visual sensitivity were estimated in the presence of heteromodal noise, whereas the sensitivity to audiovisual signals was estimated in the presence of heteromodal signals. Fidell thus tested for crossmodal, not attentional, effects.

Given certain assumptions, estimates can be made of the expected impact of stimulus uncertainty. Mulligan and Shaw (1981) used this approach to study auditory and visual localization. Data from the unimodal conditions provided worst-case estimates of localization in the bimodal condition, assuming that attentional effects were absent and only stimulus uncertainty affected performance. Actual performance in the bimodal condition did not fall below this lower bound, suggesting that the results could be explained entirely in terms of stimulus uncertainty. However, it is also possible that the effects of stimulus uncertainty were overestimated, and that performance in the bimodal condition was, in fact, constrained by attentional limitations.

Even if this were not the case, compound multimodal tasks provide no indication of the degree to which divided attention affects each modality. If performance declines in the bimodal condition, it is not clear whether this decline stems from impaired functioning in vision, audi- tion, or both. It is even conceivable (though perhaps not likely) that divided attention impairs one modality, but improves another, so that no net effect is found. Compound tasks are therefore unsuitable for studying attentional effects in multimodal perception. Similar considerations led Garner and Morton (1969) to argue vehemently against their use in studies of unimodal perception.

\section{Concurrent Tasks}

Concurrent multimodal tasks avoid all of these problems. They yield separate performance measures, as well as separate estimates of the attentional effect, for each modality in the bimodal condition. They also eliminate the need for observers to combine judgments from separate modalities, thereby avoiding the stimulus uncertainty problem.

Experiments that utilize concurrent tasks typically incorporate the same three attentional conditions found in compound tasks. The difference is that, in the bimodal condition, concurrent tasks require a separate response for each modality, whereas compound tasks require only a single response.

Concurrent tasks can test for both crossmodal and attentional effects in a single design, provided that heteromodal signals are presented in all three conditions (Figure 5). The basic elements of this procedure have already been discussed. The detectability of an auditory signal in the presence of visual noise is found by comparing the responses to $A_{N} V_{N}$ with those to $A_{S} V_{N}$. The detectability of an auditory signal in the presence of a visual signal is found by comparing the responses to $A_{N} V_{S}$ with those to $A_{S} V_{S}$. These measures are computed separately for the bimodal and unimodal auditory conditions. Crossmodal effects are implicated if, in either condition, the detectability of an auditory signal given visual noise differs

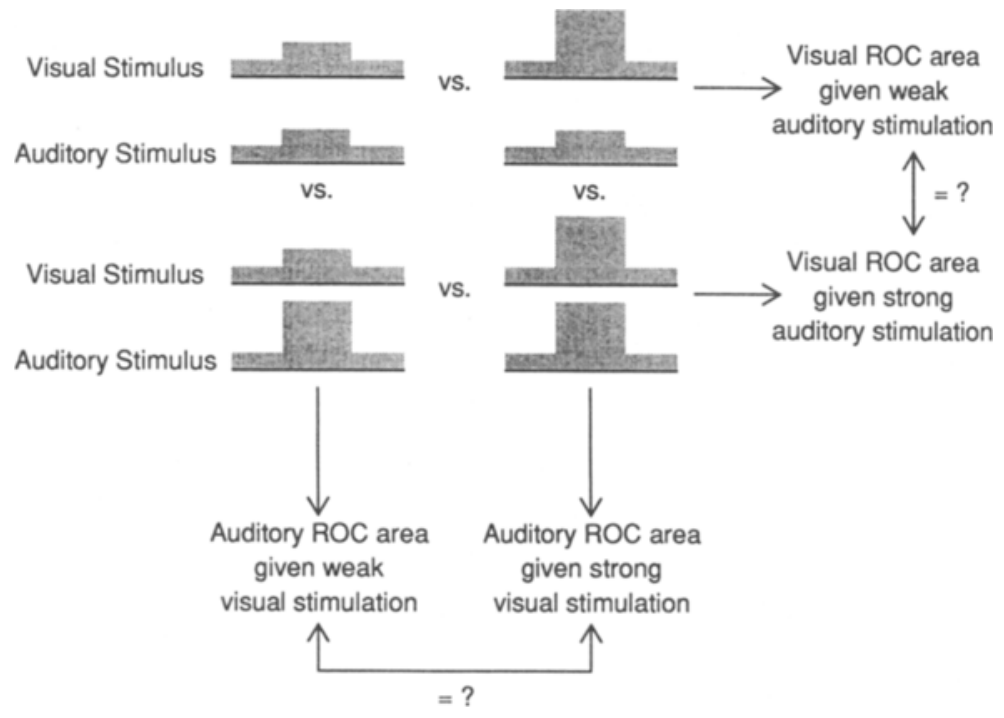

Figure 5. Stimulus pairs and receiver-operating characteristic (ROC) areas for the bimodal condition of a concurrent discrimination task. Each unimodal condition utilizes the same four stimulus pairs, but yields ROC areas for only that modality. 
from its detectability given a visual signal. Attentional effects are implicated if, given either visual noise or a visual signal, the detectability of an auditory signal differs between the unimodal and bimodal conditions. An interaction between crossmodal and attentional effects is implicated if the magnitude of the crossmodal effect varies with the attentional condition. A similar series of comparisons is made to check for crossmodal and attentional effects on visual perception.

Suprathreshold stimuli should be used on both signal and noise trials, to eliminate the temporal uncertainty problem. This changes the task from detection to discrimination, which is evident by comparing Figures 3 and 5 . In the top portion of Figure 3, noise trials lack overt visual stimulation, whereas in the top portion of Figure 5, noise trials present low-intensity visual stimuli that observers must distinguish from high-intensity visual stimuli. Laming (1985) noted that the psychometric functions for detection and discrimination typically have different shapes. However, our interest is in whether the function changes with changes in heteromodal stimulation, which can be tested in detection or discrimination tasks (although different tasks may yield different results if crossmodal effects vary with the intensity of heteromodal stimulation).

One way to implement concurrent tasks is to have observers in the bimodal condition make a single response that reports on both modalities simultaneously. For example, after each trial in a yes/no signal detection task, observers can verbally respond " $n$ one" to $A_{N} V_{N}$, " $a u$ ditory" to $A_{S} V_{N}$, "visual" to $A_{N} V_{S}$, and "both" to AsVs.

This method was used by Eijkman and Vendrik (1965) and by Loveless et al. (1970, Experiment 2), who found no evidence for attentional effects when they asked subjects to detect tones and/or flashes of light. Unfortunately, neither study presented heteromodal signals in the unimodal conditions. Since the bimodal condition involved all four stimulus pairs, the attentional and crossmodal manipulations were confounded. Furthermore, $A_{N}$ and $V_{N}$ were set at zero in both studies, so that the unimodal and bimodal conditions may have differed in temporal uncertainty. Finally, stimuli were presented for $1 \mathrm{sec}$, which may have reduced attentional effects by allowing observers to switch their attention from one modality to another during stimulus presentation. This explanation draws support from Massaro and Warner (1977, Experiment 1), who used briefer stimuli and found evidence for attentional effects when subjects were asked to discriminate high- and low-frequency tones, and visual presentations of the letters " $U$ "' and "V." This result could indicate merely that subjects find simultaneous reporting difficult, and err in combining the two responses. However, the attentional effect persisted when simultaneous reporting was required in the unimodal and the bimodal conditions.

Simultaneous reporting is suitable for 2AFC or yes/no signal detection tasks, since the bimodal condition then involves only four response alternatives. However, more sophisticated designs (e.g., rating tasks with three or more response categories) cannot use simultaneous reporting because the number of response alternatives in the bimodal condition is excessive. In these designs, observers must make their two responses in the bimodal condition serially.

Serial reporting requires two motor responses on bimodal trials, as opposed to only one on unimodal trials. The effects of motor activity on performance are not clear, but potential biases can be eliminated by having observers make two responses per trial in all conditions. (The two responses in the unimodal conditions may be identical, in which case one or the other can be ignored.)

Serial reporting in the bimodal condition also requires one judgment to be held in memory while the other is reported. This imposes seriality, even if the two judgments can be made in parallel. However, serial reporting should affect only the response that is held in memory, which is presumably the second one. Thus, if the attentional effect is an artifact of serial reporting, it should be limited to a single modality and should switch modalities as observers reverse their response order in the bimodal condition. Tulving and Lindsay (1967) found such an effect when they asked observers to detect auditory and visual signals of varying intensity: Performance in the bimodal condition declined when the heteromodal response preceded the homomodal response. However, a true attentional effect was also found: Unimodal exceeded bimodal performance in both modalities, regardless of the response order.

If serial reporting is problematic, observers can report on only one modality in the bimodal condition, as indicated by a posttrial cue (Sperling, 1960). Forbes, Taylor, and Lindsay (1967) used this method in a study involving two auditory and two visual discrimination tasks. The bimodal condition required observers to perform all four tasks simultaneously. A visual cue then indicated on which task the observer should report. However, attentional effects were observed even when the cue preceded the test stimuli by $4 \mathrm{sec}$, which suggests that the cue interfered with performance.

Serial reporting can also be avoided by comparing simultaneous and successive multimodal tasks. Shiffrin and Grantham (1974) used this method to study the detection of visual, auditory, and tactile signals. A signal was presented to one modality during each trial. In the successive condition, the trials consisted of three intervals, and a given modality could be stimulated during only one of these intervals. Observers could thus selectively attend to the modality in which the signal might occur. The simultaneous condition required observers to attend to all three modalities at once, as the signal could occur in any modality at any time during a given trial. Performance was identical in the two conditions, suggesting that attentional effects were absent. A different result was obtained by Massaro and Warner (1977, Experiment 2), who used the simultaneous versus successive paradigm when they asked observers to discriminate high- and lowfrequency tones, and visual presentations of the letters 
" $U$ " and "V." Performance declined in the simultaneous condition, suggesting that an attentional effect was present.

However, the simultaneous versus successive paradigm is problematic. In both conditions, observers can optimize their performance by comparing the likelihood that Stimulus $a$ was presented in Modality $x$ with the likelihood that Stimulus $b$ was presented in Modality $y$, and selecting the modality that yields the greatest likelihood. If these likelihoods cannot be computed and converted into conveniently stored metrics during each interval of the successive trials, the successive condition will involve simultaneous processing. The two conditions may thus impose similar attentional loads. Performance in the successive condition may also be impaired by memory limitations, just as performance in the simultaneous condition may be impaired by attentional limitations. Additional complications arise because observers may vary their criteria throughout a trial in the successive condition. A final drawback of the method is that it cannot be used to test for crossmodal effects. Since only one modality can be stimulated during any given trial, heteromodal stimulation-and thus the test for crossmodal effects-is precluded by the design.

This contrasts with concurrent multimodal tasks, which allow crossmodal and attentional effects to be tested within a single experiment. Concurrent tasks can also test for interactions between crossmodal and attentional effects. Such interactions have implications for perceptual organization. If, for example, crossmodal effects are attenuated in the bimodal condition, observers may tend to confuse stimuli in different modalities, unless they make a specific effort (as in the bimodal condition) to examine each modality separately. To date, only Shiffrin and Grantham (1974, Experiment 3) have actually checked for an interaction. They reported a reduced ability to detect auditory and visual signals in the bimodal condition, but only if heteromodal signals were present. However, this finding may have been an artifact of the response procedure: Two responses were required in the bimodal condition when a heteromodal signal was present, as opposed to only one response in the unimodal conditions, or when other stimuli were presented.

A further advantage of concurrent tasks is that they indicate how responses for the two modalities covary. This information can be used to test for correlated noise between modalities, and for the tendency of observers to make concordant or discordant judgments (see Klein, 1985 , and Olzak, 1985, for procedural details). The pattern of response covariation can also indicate whether the criteria in one modality reflect the perceived strength of stimuli in another modality (Shiffrin \& Grantham, 1974), and whether improved performance in one modality is offset by decrements in another (see Moore \& Massaro, 1973, for a unimodal example).

Clearly, concurrent paradigms can provide far more information on the mechanisms underlying multimodal perception than can other paradigms. Concurrent tasks are also economical, as they can study crossmodal and attentional effects within a single experiment. Their great explanatory power suggests that they should replace more conventional designs, although other paradigms may prove more convenient for certain applications.

\section{REFERENCES}

AshBY, F. G., \& Townsend, J. T. (1986). Varieties of perceptual independence. Psychological Review, 93, 154-179.

BenedeTTI, L. H. , Loeb, M. (1972). A comparison of auditory monitoring performance in blind subjects with that of sighted subjects in light and dark. Perception \& Psychophysics, 11, 10-16.

Bernstein, I. H. (1970). Can we see and hear at the same time? Acta Psychologica, 33, 21-35.

Bross, M. (1979). Residual sensory capacities of the deaf: A signal detection analysis of a visual discrimination task. Perceptual \& Motor Skills, 48, 187-194.

Bross, M., \& ZuBEK, J. P. (1975). Effect of auditory deprivation on visual resolving power. Canadian Joumal of Psychology, 29, 340-347.

Brown, A. E., HopkINs, H. K. (1966). Interaction of the auditory and visual sensory modalities. Journal of the Acoustical Society of America, 41, 1-6.

BuCkNer, D. N., McGrath, J. J. (1961). A comparison of performances on single and dual sensory mode vigilance tasks (Tech. Rep. No. 8). Los Angeles: Human Factors Research, Inc.

CoHen, M. M. (1981). Visual-proprioceptive interactions. In R. D. Walk \& H. L. Pick, Jr. (Eds.), Intersensory perception and sensory integration (pp. 175-215). New York: Plenum Press.

Colquhoun, W. P. (1975). Evaluation of auditory, visual, and dualmode displays for prolonged sonar monitoring in repeated sessions. Human Factors, 17, 425-437.

Craig, A., Colquhoun, W. P., \& Corcoran, D. W. J. (1976). Combining evidence presented simultaneously to the eye and the ear: $A$ comparison of some predictive models. Perception \& Psychophysics, $19,473-484$.

DASHIELL, J. F. (1939). Some rapprochements in contemporary psychology. Psychological Bulletin, 36, 1-24.

DA VENPORT, W. G. (1974). Arousal theory and vigilance: Schedules for background stimulation. Journal of General Psychology, 91, 51-59.

Davies, D. R., LANG, L., Shackleton, V. J. (1973). The effects of music and task difficulty on performance at a visual vigilance task. British Journal of Psychology, 64, 383-393.

Davis, E. T., \& Graham, N. (1981). Spatial frequency uncertainty effects in the detection of sinusoidal gratings. Vision Research, 21, $705-712$.

Dougherty, W. G., Jones, G. B., Engel, G. R. (1971). Sensory integration of auditory and visual information. Canadian Joumal of Psychology, 25, 476-485.

Egan, J. P., Greenberg, G. Z., \& Schulman, A. I. (1961). Interval of time uncertainty in auditory detection. Journal of the Acoustical Society of America, 33, 771-778.

EukmaN, E., \& Vendrik, A. J. H. (1965). Can a sensory system be specified by its internal noise? Journal of the Acoustical Society of America, 37, 1102-1109.

FIDELL, S. (1970). Sensory function in multimodal signal detection. Journal of the Acoustical Society of America, 47, 1009-1015.

Fishman, M. C., Michael, C. R. (1973). Integration of auditory information in the cat's visual cortex. Vision Research, 13, 1415-1419.

Forbes, S. M., Taylor, M. M., \& Linday, P. H. (1967). Cue timing in a multi-dimensional detection task. Perceptual \& Motor Skills, 25, $113-120$.

Garner, W. R., Morton, J. (1969). Perceptual independence: Definitions, models, and experimental paradigms. Psychological Bulletin, 72, 233-259.

GILBERT, G. M. (1941). Inter-sensory facilitation and inhibition. Journal of General Psychology, 24, 381-407.

Green, D. M., \& SweTs, J. A. (1974). Signal detection theory and psychophysics. Huntington, NY: Krieger. 
GrUber, A. (1964). Sensory alternation and performance in a vigilance task. Human Factors, 6, 3-12.

Harris, L. R., Blakemore, C., \& Donaghy, M. (1980). Integration of visual and auditory space in mammalian superior colliculus. $\mathrm{Na}$ ture, 288, 56-59.

HARTMANN, G. W. (1933). II. Changes in visual acuity through simultaneous stimulation of other sense organs. Journal of Experimental Psychology, 16, 393-407.

KINCHLA, R. A. (1969). Temporal and channel uncertainty in detection: A multiple observation analysis. Perception \& Psychophysics, $5,129-136$.

Klayman, B. E. (1973a). Detection and the timing of a heteromodal cue: Auditory-visual interaction. Joumal of Psychology, 83, 201-213.

KLAYMAN, B. E. (1973b). Heteromodal cueing and auditory-visual interaction: A literature review. Joumal of Psychology, 83, 173-199.

KLEIN, S. (1985). Double-judgement psychophysics: Problems and solutions. Journal of the Optical Society of America A: Optics and Image Science, 2, 1560-1585.

KNUDSEN, E. I. (1983). Early auditory experience aligns the auditory map of space in the optic tectum of the barn owl. Science, 222, 939-942.

KNUdSEN, E. I., \& KNUdSEN, P. F. (1985). Vision guides the adjustment of auditory localization in young barn owls. Science, 230, 545-548.

Kravkov, S. W. (1930). Über die Abhängigkeit der Sehschärfe vom Schallreiz. Archiv fur Ophthalmologie, 124, 334-338.

Laming, D. (1985). Some principles of sensory analysis. Psychological Review, 92, 462-485.

LAwSON, K. R., \& TuRKEWITZ, G. (1980). Intersensory function in newborns: Effect of sound on visual preference. Child Development, 51, 1295-1298.

LEWKowicz, D. J., \& TuRKewitz, G. (1981). Intersensory interaction in newborns: Modification of visual preferences following exposure to sound. Child Development, 52, 827-832.

LoNDON, I. D. (1954). Research on sensory interaction in the Soviet Union. Psychological Bulletin, 51, 531-568.

Loveless, N. E., Brebner, J., \& Hamilton, P. (1970). Bisensory presentation of information. Psychological Bulletin, 73, 161-199.

Maier, B., Bevan, W., \& Behar, I. (1961). The effect of auditory stimulation upon the critical flicker frequency for different regions of the visual spectrum. American Joumal of Psychology, 74, 67-73.

MALONEY, D. M., \&ELCh, R. B. (1972). The effect of accessory auditory stimulation upon detection of visual signals. Psychonomic Science, 29, 345-347.

Marks, L. E. (1975). On colored-hearing synesthesia: Cross-modal translations of sensory dimensions. Psychological Bulletin, 82, 303-331.

MARKs, L. E. (1978). The unity of the senses: Interrelations among the modalities. New York: Academic Press.

MaruYama, K. (1959). The effect of intersensory tone stimulation on absolute light threshold. Tohoku Psychologica Folia, 17, 51-81.

Massaro, D. W., \& Warner, D. S. (1977). Dividing attention between auditory and visual perception. Perception \& Psychophysics, 21, 569-574.

MCGrATH, J. J. (1963). Irrelevant stimulation and vigilance performance. In D. N. Buckner \& J. J. McGrath (Eds.), Vigilance: A symposium (pp. 3-19). New York: McGraw-Hill.

McGrath, J. J. (1965). Performance sharing in an audio-visual task. Human Factors, 7, 141-153.

Meredith, M. A., \& STeIN, B. E. (1983). Interactions among converging sensory inputs in the superior colliculus. Science, 221, 389-391.

Meredith, M. A., \& Stein, B. E. (1985). Descending efferents from the superior colliculus relay integrated multisensory information. Science, 227, 657-659.

MOORE, J. J., MASSARo, D. W. (1973). Attention and processing capacity in auditory recognition. Journal of Experimental Psychology, 99, 49-54.

MORRELL, F. (1965). Clinical neurology: Some applications of scanning by computer. California Medicine, 103, 406-416.
Mulligan, R. M., Shaw, M. L. (1981). Attending to simple auditory and visual signals. Perception \& Psychophysics, 30, 447-454. Newman, E. A., \& HaRTune, P. H. (1981). Integration of visual and infrared information in bimodal neurons of the rattlesnake optic tectum. Science, 213, 789-791.

OlzaK, L. (1985). Interactions between spatially tuned mechanisms: Converging evidence. Journal of the Optical Society of America A: Optics and Image Science, 2, 1551-1559.

Osborn, W. C., Sheldon, R. W., \& BAKER, R. A. (1963). Vigilance performance under conditions of redundant and nonredundant signal presentation. Journal of Applied Psychology, 47, 130-134.

RYAN, T. A. (1940). Interrelations of the sensory stimuli in perception. Psychological Bulletin, 37, 659-698.

SCHULTZ, D. P. (1965). Sensory restriction: Effects on behavior. New York: Academic Press.

Shiffrin, R. M., \& Grantham, D. W. (1974). Can attention be allocated to sensory modalities? Perception \& Psychophysics, 15, 460-474.

SHIPLEY, T. (1970). Evoked brain potentials and sensory interaction in the retarded child. American Journal of Mental Deficiency,74, 517-523.

SPERLING, G. (1960). The information available in brief visual presentations. Psychological Monographs, 74(11, Whole No. 498).

SPERLING, G., Dosher, B. A. (1986). Strategy and optimization in human information processing. In K. Boff, L. Kaufman, \& J. P. Thomas (Eds.), Handbook of perception and human performance: Vol. 1. Sensory processes and perception (chap. 2). New York: Wiley.

STEIN, B. E., \& ARIGBEde, M. O. (1972). Unimodal and multimodal response properties of neurons in the cat's superior colliculus. Experimental Neurology, 36, 179-196.

TAYLOR, R. L. (1974). An analysis of sensory interaction. Neuropsychologia, 12, 65-71.

TAYloR, R. L., \& CAMPBEll, G. T. (1976). Sensory interaction: Vision is modulated by hearing. Perception, 5, 467-477.

TULVING, E., LINDSAY, P. H. (1967). Identification of simultaneously presented simple visual and auditory stimuli. Acta Psychologica, 27, 101-109.

Tzelgov, J., Srebro, R., Henik, A., Kushelevsky, A. (1987). Radiation search and detection by ear and by eye. Human Factors, 29, 87-95.

WALK, R. D., \& Pick, H. L., JR. (EDs.). (1981). Intersensory perception and sensory integration. New York: Plenum Press.

WATkINS, W. H. (1964). Effect of certain noises upon detection of visual signals. Journal of Experimental Psychology, 67, 72-75.

W ATKINS, W. H., FeEHRER, C. E. (1965). Acoustic facilitation of visual detection. Journal of Experimental Psychology, 70, 332-333.

WELCH, R. B., \& WARREN, D. H. (1980). Immediate perceptual response to intersensory discrepancy. Psychological Bulletin, 88, 638-667.

WiCKelgren, B. G. (1971). Superior colliculus: Some receptive field properties of bimodally responsive cells. Science, 173, 69-72.

ZerTZ, K. (1931). Gegenseitige Beeinflussung von Farb- und Tonerlebnissen. Zeitschrift für Psychologie, 121, 257-356.

Zentall, S. S., Zentall, T. R. (1983). Optimal stimulation: A model of disordered activity and performance in normal and deviant children. Psychological Bulletin, 94, 446-471.

ZUBEK, J. P. (1969). Sensory and perceptual-motor processes. In J. P. Zubek (Ed.), Sensory deprivation: Fifteen years of research (pp. 207253). New York: Appleton-Century-Crofts.

\section{NOTE}

1. Homomodal stimuli are usually called "primary," whereas heteromodal stimuli are called "secondary"' or "accessory." This implies that homomodal stimuli are more salient or important than heteromodal stimuli. However, the experimenter's intention in this respect is not always realized: Heteromodal and homomodal stimuli sometimes provide comparable amounts of information. It therefore seems prudent to avoid using terms such as "primary" and "secondary."

(Manuscript received November 18, 1987; revision accepted for publication June 27,1988 .) 